\title{
Dynamic Linear Combination of Two-Class Classifiers
}

\author{
Carlo Lobrano ${ }^{1}$, Roberto Tronci ${ }^{1,2}$, Giorgio Giacinto ${ }^{1}$, and Fabio Roli ${ }^{1}$
}

1 DIEE Dept. of Electrical and Electronic Engineering, University of Cagliari, Italy

2 Laboratorio Intelligenza d'Ambiente, Sardegna DistrICT, Sardegna Ricerche, Italy c.lobrano@gmail.com, \{roberto.tronci,giacinto, roli\}@diee.unica.it

\begin{abstract}
In two-class problems, the linear combination of the outputs (scores) of an ensemble of classifiers is widely used to attain high performance. In this paper we investigate some techniques aimed at dynamically estimate the coefficients of the linear combination on a pattern per pattern basis. We will show that such a technique allows providing better performance than those of static combination techniques, whose parameters are computed beforehand. The coefficients of the linear combination are dynamically computed according to the Wilcoxon-Mann-Whitney statistic. Reported results on a multi-modal biometric dataset show that the proposed dynamic mechanism allows attaining very low error rates when high level of precision are required.
\end{abstract}

Keywords: Classifier ensembles, two-class classification, biometric systems.

\section{Introduction}

Many applications, such as anomaly detection, biometric authentication, etc., require the design of classifiers that discriminate one class of objects (a.k.a. the positive class) from all other objects (a.k.a. the negative class). This is usually attained by measuring how similar the sample is with respect to the positive class, and classifying the pattern as positive if the similarity score is above some predefined threshold. The performance of this kind of classifiers is evaluated by the trade-off between misclassification errors at some significant threshold values, or by resorting to threshold-independent measures such as the Area Under the ROC Curve (AUC) [1]. However, in security applications, the performances are usually evaluated for a limited range of threshold values, corresponding to very low error rates. Unfortunately, the required performance for security applications are hardly met by any individual classifiers.

To improve the overall performance with respect to the selection of the "best" single classifier, the approaches based on ensemble of classifiers are widely used [677. On one hand, the selection of the "best" classifier is not a trivial task, and is highly dependent on the criteria used to rank the classifiers. On the other hand, the use of an ensemble of classifiers allows exploiting the complementary discriminatory information that all the ensemble members may encapsulate. When 
the classifiers produce similarity scores, the combination is generally performed at the score level by producing a new score according to some "fusion" rule (e.g., by a linear combination) 7/12. So far, most of the solutions presented in the literature adopts a "static" approach, in the sense that the parameters of the combination rule do not depend on the sample to be classified. It is easy to see that further improvements are expected if the combination rule makes use of sample-specific parameters [16/2910]. For instance, a larger degree of separation between the distributions of positive and negative samples can be attained by dynamically tuning the combination parameters according to an estimation of the probability that the sample belongs to the positive class [13.

In this paper, we propose a novel dynamic combination strategy. For each pattern, and for each classifier, we propose the computation of an index called Score Decidability Index (SDI) that is based on the Wilcoxon-Mann-Whitney statistic (WMW). Then, the coefficients of the linear combination are computed as a function of these indexes. This index measures, for each classifier, and for each pattern, the confidence in classifying the pattern either as positive or negative, according to the score assigned to that pattern, and to the scores assigned to a reference set made up of positive and negative.

The SDI can also be seen as a different representation of the original score assigned by each classifier to a given pattern, as it represents the likelihood with which the original score is drawn from either the positive or negative distributions of scores. A new fused score can then be computed by averaging the SDI values. Finally, the SDI values produced by the ensemble of classifiers for a given pattern can be further used to compute the coefficients of a simplified combination rule, where only two of the scores produced by the ensemble are used, namely the maximum and the minimum score.

Section 2 presents the Score Decidability Index (SDI), while its use to compute the coefficients of the dynamic linear combination is presented in Section 3. Section 4 illustrates other uses of the SDI to produce a new transformed score, and the coefficients of simplified dynamic combination. Section 5 shows the experimental results on a multi-modal dataset, where the effectiveness of the proposed techniques are outlined.

\section{Score Decidability Index}

Usually, the parameters (weights) for a linear combination of outputs produced by an ensemble of two-class classifiers are computed through some estimations or measurements performed on the data. One way to compute these parameters is to exploit one (or more) performance measure or statistic. In this paper we propose the use of a measure called Score Decidability Index to estimate the parameters of a dynamic linear combination. This index will be derived from the Wilcoxon-Mann-Whitney (WMW) statistic [4].

Let us consider a two-class problem, where the two classes are denoted as positive $\left(\omega_{+}\right)$and negative $\left(\omega_{-}\right)$. For each pattern $x_{i}$ to be classified, a two-class classifier $C_{k}$ usually produce an output score $s_{i k}=f_{k}\left(x_{i}\right)$. Then, a decision 
threshold th is set, and patterns whose score is greater than the threshold are assigned to the positive class, otherwise they are assigned to the negative class.

Let now us consider a set of patterns whose class is known for a generic classifier $C_{k}$, and let:

$$
\begin{aligned}
& S_{k}^{+}=\left\{s_{i k}^{+}=f_{k}\left(x_{i}\right) \mid x_{i} \in \omega_{+}\right. \\
& S_{k}^{-}=\left\{\begin{array}{l}
, \\
s_{i k}^{-}=f_{k}\left(x_{i}\right) \mid x_{i} \in \omega^{-}
\end{array}\right\}, \quad \forall i
\end{aligned}
$$

The performance of two-class classifiers $C_{k}$ for all possible values of the decision threshold th can be summarized by the AUC, whose value can be computed by resorting to the WMW statistic [5]:

$$
A U C_{k}=\frac{\sum_{i=0}^{n_{+}} \sum_{j=0}^{n_{-}} I\left(s_{i k}^{+}, s_{j k}^{-}\right)}{n_{+} \cdot n_{-}}
$$

where $n_{+}$and $n_{-}$represent the number of positive and negative patterns, and the function $I(a, b)$ is equal to 1 if $a>b$, otherwise it is equal to 0 . This formulation of the AUC can be also seen as a measure of the probability that the classifier ranks a randomly chosen positive sample higher than a randomly chosen negative sample, i.e. $P\left(S^{+}>S^{-}\right)$[4].

Let us define

$$
\begin{aligned}
& r_{-}(s)=\frac{\sum_{i=0}^{n_{+}} I\left(s_{i}^{+}, s\right)}{n_{+}} \simeq P\left(S^{+}>s\right) \\
& r_{+}(s)=\frac{\sum_{j=0}^{n_{-}} I\left(s, s_{j}^{-}\right)}{n_{-}} \simeq P\left(s>S^{-}\right)
\end{aligned}
$$

Hence $r_{-}(s)$ represents the probability that the score $s$ is lesser than a score coming from the positive distribution, and $r_{+}(s)$ represents the probability that the score $s$ is larger than a score coming from the negative distribution.

It can be easily seen that the WMW statistic in Eq. (1) can be written in either of the two following formulations:

$$
\frac{\sum_{i=0}^{n_{+}} \sum_{j=0}^{n_{-}} I\left(s_{i k}^{+}, s_{j k}^{-}\right)}{n_{+} \cdot n_{-}} \rightarrow\left\{\begin{array}{l}
\frac{\sum_{j=0}^{n_{-}} r_{-}\left(s_{j k}^{-}\right)}{n_{-}} \\
\frac{\sum_{i=0}^{n_{+}} r_{+}\left(s_{i k}^{+}\right)}{n_{+}}
\end{array}\right.
$$

Thus, $r_{+}(s)$ and $r_{-}(s)$ represent an estimation of the contribution of the score $s$ to the value of the AUC in the case it belongs either to the positive or negative class, respectively. Given the sets of scores $S_{k}^{+}$and $S_{k}^{-}$produced by a two-class classifier $C_{k}$ on a training set, for each score $s_{i k}$ related to a test pattern $x_{i}$, the Score Decidability Index (SDI) can be defined as

$$
\Delta\left(s_{i k}\right)=r_{+}\left(s_{i k}\right)-r_{-}\left(s_{i k}\right)
$$

that is related to the likelihood the pattern $x_{i}$ is drawn either from the positive or negative distributions of scores. If $\Delta\left(s_{i k}\right)=1$ (i.e., $P\left(s_{i k}>S_{k}^{-}\right)=1$ and 
$\left.P\left(S_{k}^{+}>s_{i k}\right)=0\right)$, the score $s_{i k}$ is larger than any other score in the training set $S_{k}^{+} \bigcup S_{k}^{-}$. As a consequence, it is more likely that pattern $x_{i}$ comes from the positive rather than from the negative distribution. Similarly, if $\Delta\left(s_{i k}\right)=-1$ (i.e., $P\left(s_{i k}>S_{k}^{-}\right)=0$ and $\left.P\left(S_{k}^{+}>s_{i k}\right)=1\right)$, the score $s_{i k}$ is lesser than any other score in the training set, so that it is more likely that the sample comes from the negative distribution. The other values of $\Delta\left(s_{i k}\right)$ in the range $[-1,1]$ accounts for the uncertainty in the classification of the sample whose score is $s_{i k}$, the larger the value of $\left|\Delta\left(s_{i k}\right)\right|$, the more confident is the class decision.

\section{Dynamic Linear Combination}

As stated in the Introduction, the linear combination of scores is one of the most widely used way to fuse outputs from different classifiers.

$$
s_{i}^{l c}=\sum_{k=1}^{\mathbf{N}} \alpha_{k} \cdot s_{i k}
$$

Usually, some constraints are introduced to simplify the parameters estimation. For example, affine combinations are obtained if $\sum_{k} \alpha_{k}=1$, conical combinations are those combinations for which $\alpha_{k} \geq 0$, and convex or weighted combinations require that $\alpha_{k} \geq 0$, and $\sum_{k} \alpha_{k}=1$.

One of the simplest form of linear combination is obtained by averaging the outputs of the classifiers (a.k.a. the Mean-rule). This rule implicitly assume that all the classifiers are assigned the same weight [14. However, it has been pointed out that a weighted combination outperforms the Mean-rule when the classification problem is made up of imbalanced classes [3]. The weights of the combination are usually computed by maximizing a measure of performance on a training set. It is worth noting that usually each classifier is assigned a unique weight that does not depend on the sample to be classified. In other words, typically the weighted combination aims at improving the average performance of the classification system. Moreover, the optimization algorithm may exhibit a high computational cost, depending on the heuristic used to maximize the selected performance measure or statistic [8,15. On the other hand, it is easy to see that an optimal linear combination rule should require weights that depends both on each individual classifier, and on the pattern to be classified.

$$
s_{i}^{*}=\sum_{k=1}^{\mathbf{N}} \alpha_{i k} \cdot s_{i k}
$$

Such techniques are usually called "dynamic" combination techniques. However, if it is difficult and computational costly to estimate the optimal set of weights for each individual classifier, the estimation of dynamic weights may result in a more complex problem [7]. Usually this problem is solved by estimating the behavior of each classifier in the region containing the test sample. Different heuristics have been proposed that are based on different definitions of classifier behavior, and different definition of regions. 
In this paper, we propose to exploit the SDI formulated in Section 2 to derive the weights of the combination so that the distributions of the combined output for the two classes allows effective separation. One way to achieve this goal is to provide large weights for each score related to positive samples, so that the combined score is as high as possible, and to provide small weights for each score related to negative samples, so that the combined score is as small as possible. Actually, each classifier in the ensemble can provide the information on the most likely class a test pattern belongs to. If a set of reference patterns related to the positive and negative classes are available, the distribution of the outputs on such a set can be representative of the behavior of that classifier for the two classes. Thus, if we compare the output $s$ produced by each classifier in the ensemble with the outputs of the same classifier on the reference set, that classifier supports the following conclusions

if $s>s_{i k}, \forall s_{i k} \in S_{k}^{-}$, then the pattern is likely to be positive if $s<s_{i k}, \forall s_{i k} \in S_{k}^{+}$, then the pattern is likely to be negative

For any other intermediate case, the classifier may support one decision or the other with different strength, depending on the fractions of the reference set which support the two above propositions.

Actually the value of SDI can be used to compute the weights of the linear combination, as for each classifier and for each score it can provide the information on the most likely class, and the strength of the decision. It can be easily seen that the sign of $\Delta$ indicates the most likely class, while the modulus of $|\Delta|$ is a measure of the "strength" of the class prediction. Thus we propose to use the SDI to compute the weights of a dynamic linear combination as:

$$
\alpha_{i k}=\frac{\Delta\left(s_{i k}\right)+1}{2}
$$

where the value of $\alpha_{i k}$ is in the range $[0,1]$ in agreement with the normalization used for the outputs of the classifier. We will refer to this technique as DLC.

\section{Other Dynamic Rules Based on Score Decidability Index}

The rationale behind the computation of the weights for the linear combination shown in the previous section may give rise to other combination rules.

\subsection{The Score Decidability Index as a normalized Score}

In the previous section we claimed that for each pattern and for each classifier, the sign of $\Delta$ indicates the most likely class, while the modulus of $|\Delta|$ is a measure of the "strength" of the class prediction. If we normalize $\Delta$ in the range [0, 1$]$, the resulting value can be used as a new normalized score for each classifier. Then, these new values can be combined by any combination mechanism. In order to keep the system simple, and in account of the meaning of these new normalized 
scores, we propose to average these new values. We will refer to this technique as SDI mean:

$$
s_{i}^{*}=\frac{1}{N} \sum_{k=1}^{N} \frac{\Delta_{i k}+1}{2}
$$

\subsection{Simplified Score Combination}

The Score Decidability Index can be also used in the framework of a simplified combination scheme called Dynamic Score Combination (DSC) [13]. Two similar formulations of the DSC have been proposed:

$$
\begin{aligned}
& s_{i}^{*}=\beta_{1 i} \cdot \max _{k}\left(s_{i k}\right)+\beta_{2 i} \cdot \min _{k}\left(s_{i k}\right) \\
& s_{i}^{*}=\beta_{i} \cdot \max _{k}\left(s_{i k}\right)+\left(1-\beta_{i}\right) \cdot \min _{k}\left(s_{i k}\right)
\end{aligned}
$$

In Eq.(10) the two parameters $\beta_{1 i}$, and $\beta_{2 i}$ "estimate" the likelihood of $x_{i}$ being a positive or a negative pattern. Eq.(11) is similar to Eq.(10), where the constraints $\beta_{2 i}=\left(1-\beta_{1 i}\right)$ and $\beta_{i} \in[0,1]$ are added. DSC basically combines only two values among all the scores produced by the ensemble of classifiers, namely the smallest and the biggest values. On the other hand, the behavior of the ensemble (i.e., all the scores produced by the ensemble) is used to compute the values of the $\beta \mathrm{s}$. In the following we propose two different methods to embed the SDI into Eq.s(10), and (11).

Dynamic Score Combination by $\Delta$ voting. Let us consider the formulation of DSC in Eq.(10) where the values of $\beta$ are continuous. By taking into account that the decidability of the class of the sample is critical if the value of $\Delta$ is close to zero, we can fuse the SDI of the ensemble of classifiers by a Voting mechanism. In particular, we evaluate the "likelihood" of the sample belonging either to the positive or the negative class, by counting the fraction of the classifiers that exhibit a decidability index larger than an offset $\alpha$ :

$$
\begin{aligned}
& \beta_{1 i}=\frac{1}{N} \sum_{k=1}^{N} I\left(\Delta\left(s_{i k}\right), \alpha\right) \\
& \beta_{2 i}=\frac{1}{N} \sum_{k=1}^{N} I\left(-\Delta\left(s_{i k}\right), \alpha\right)
\end{aligned}
$$

Typical values of $\alpha$ are $0.05,0.1,0.15$, and 0.2 . The reported experimental results are related to $\alpha=0.05$.

Dynamic Score Combination by $\boldsymbol{\Delta}$ mean. In this case, we take into account the formulation of the DSC reported in Eq.(11). In this case, the values of $\Delta$ can be used to compute the parameter $\beta_{i}$ by taking into account the average and the standard deviation of $\Delta$ among all the classifiers as follows:

$$
\begin{aligned}
\Delta^{*}\left(\mathbf{s}_{i}\right) & =\frac{\frac{1}{N} \sum_{k=1}^{N} \Delta\left(s_{i k}\right)}{\sigma_{\Delta\left(s_{i k}\right)}} \\
\beta_{i} & =\frac{1}{1+e^{-\gamma \cdot \Delta^{*}\left(\mathbf{s}_{i}\right)}}
\end{aligned}
$$


where the sigmoid in the Eq (14) is used to normalize the value of $\Delta^{*}\left(\mathbf{s}_{i}\right)$ in the range $[0,1]$. Typical values of $\gamma$ in the normalization process are from 1 to 6 . The reported experimental results are related to $\gamma=3$.

\section{Experimental Results}

The experiments have been performed on the Biometric Authentication Fusion Benchmark Database (BA-Fusion), a multi-modal database of similarity scores artificially created from experiments carried out on the XM2VTS face and speaker verification database [11. This dataset contains similarity scores from 8 classifiers, and the scores have been normalized by the Tanh rule [12.

Reported experiments aim at assessing the performance of the proposed techniques in terms of different performance measures. In particular, the AUC, the EER, have been used, as well as error measures at four operating points that are generally used to test security systems, namely FPR 1\%, FPR 0\%, FNR 1\% and FNR 0\%. Thus, the FNR (FPR) attained when the FPR (FNR) is equal to $1 \%$ or $0 \%$ are measured, respectively.

Experiments have been carried out by creating ensembles where the number of classifier in the ensemble ranges from 2 to 8 . In this way, we create ensembles that contain all possible subsets of classifiers from the original pool of 8 classifiers. In order to get unbiased results, a 4-fold cross-validation technique has been used. The dataset has been subdivided into 4 subsets, so that one subset at a time was used for training, while the other three have been used for testing. Results are reported in terms of average and standard deviation over the four trials, and over all the possible ensemble of classifiers for a given ensemble size.

The performance of the proposed algorithms have been compared to those of the Mean-rule, as this is a simple and effective way of combining multiple scores. Very often experimental results show that the Mean-rule provides significant performance improvements not only with respect to individual classifiers, but also with respect to other combination rules. Performance are also compared to the best performance provided by the individual classifiers included in the ensemble. It is worth noting that for each measure of performance, the best value can be related to a different classifier in the ensemble.

Results reported in Fig 1 show that the average performance improve as the size of the ensemble increases. This results shows that the proposed combination mechanisms allow exploiting the complementary information that the individual classifiers may exhibit. In particular, the combination of classifiers always allows outperforming the best classifier, and provide very low error rates. By inspecting the figure, an ensemble size equal to five can be a good compromise between performance and ensemble complexity. For this reason, Table 1 shows the detailed numerical results in terms of the average and standard deviation for an ensemble size equal to five.

Fig. 1(a) shows the results in terms of the AUC. It is easy to see that all the combination methods provide very high AUC values, very close to each other. Fig.s 1(b)-(d) show the performance in terms of EER and FPR 1\%, respectively. 


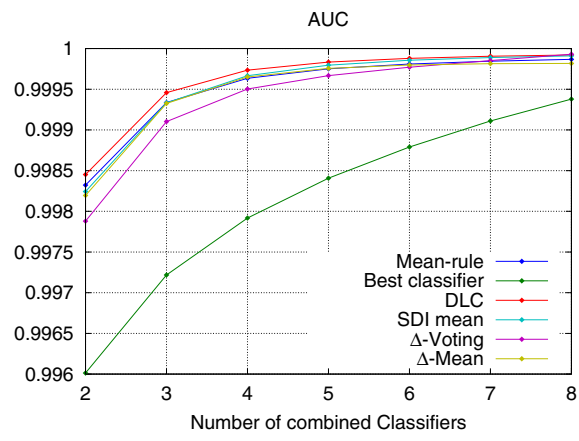

(a)

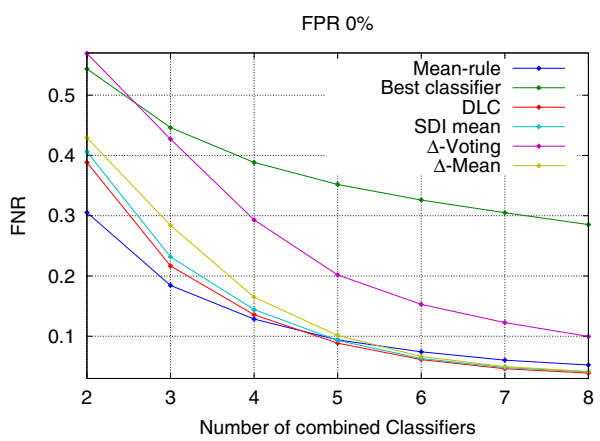

(c)

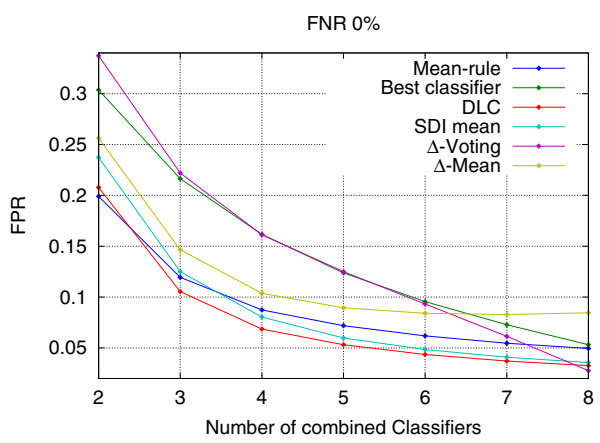

(e)

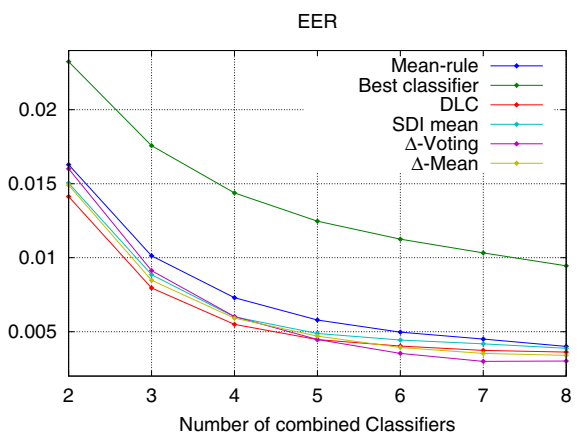

(b)

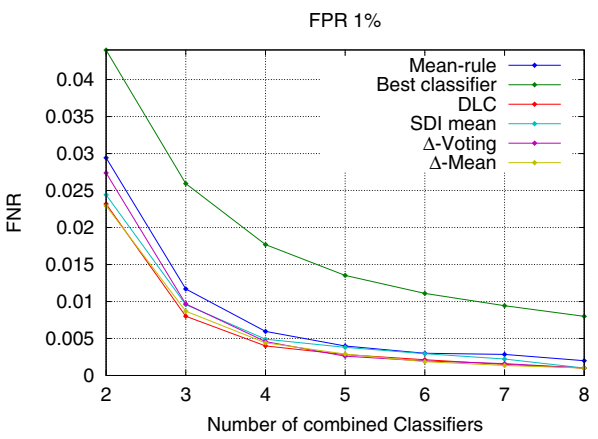

(d)

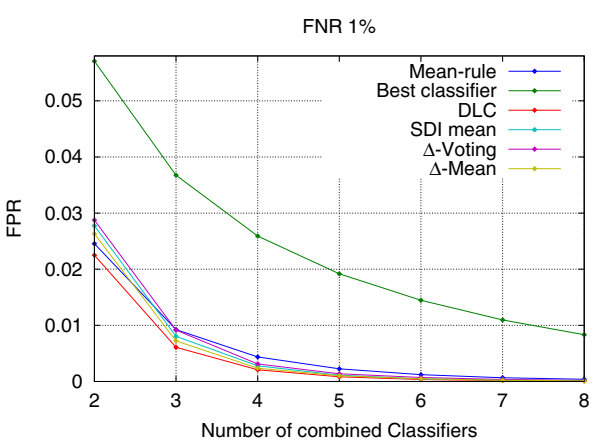

(f)

Fig. 1. Average performance for each ensemble size 
Table 1. Performance in terms of average and standard deviation (between brackets) for all the ensembles of 5 classifiers. Results with a $\circ$ indicate that the difference in performance from those achieved by the Mean-rule are not statistically significant according to the t-test with a $95 \%$ confidence. The best performance are in italics.

\section{AUC EER}

\begin{tabular}{lcc}
\hline Mean-rule & $0.9998( \pm 0.0002)$ & $0.0058( \pm 0.0019)$ \\
Best classifier & $0.9984( \pm 0.0014)$ & $0.0125( \pm 0.0046)$ \\
\hline DLC & $0.9998( \pm 0.0002)$ & $0.0045( \pm 0.0017)$ \\
SDI mean & $0.9998( \pm 0.0002)$ & $0.0049( \pm 0.0023)$ \\
\hline$\Delta$ Voting & $0.9997( \pm 0.0005)$ & $0.0045( \pm 0.0021)$ \\
$\Delta$ mean & $\circ 0.9998( \pm 0.0003)$ & $0.0047( \pm 0.0019)$ \\
\hline
\end{tabular}

\section{FPR-0\%}

FPR-1\%

FNR-1\%

FNR-0\%

\begin{tabular}{lcccc}
\hline Mean-rule & $0.0941( \pm 0.0342)$ & $0.0040( \pm 0.0026)$ & $0.0023( \pm 0.0017)$ & $0.0719( \pm 0.0827)$ \\
Best classifier & $0.3518( \pm 0.1148)$ & $0.0135( \pm 0.0092)$ & $0.0192( \pm 0.0181)$ & $0.1237( \pm 0.1120)$ \\
\hline DLC & $0.0886( \pm 0.0469)$ & $0.0028( \pm 0.0024)$ & $0.0008( \pm 0.0010)$ & $0.0532( \pm 0.0599)$ \\
SDI mean & $\circ 0.0931( \pm 0.0455)$ & $0.0038( \pm 0.0029)$ & $0.0011( \pm 0.0020)$ & $0.0598( \pm 0.0619)$ \\
\hline$\Delta$ Voting & $0.2017( \pm 0.1622)$ & $0.0026( \pm 0.0024)$ & $0.0014( \pm 0.0013)$ & $0.1250( \pm 0.1981)$ \\
$\Delta$ mean & $\circ 0.1015( \pm 0.0723)$ & $0.0029( \pm 0.0025)$ & $0.0010( \pm 0.0013)$ & $0.0895( \pm 0.1150)$ \\
\hline
\end{tabular}

Regardless the ensemble size, all the proposed methods outperform those of the mean rule. However, when the EER is considered, the DLC outperform all other measures for ensemble sizes smaller than or equal to five, while $\Delta$-voting provides the best performance for sizes greater than five. On the other hand, when the FPR $1 \%$ is considered, the DLC provides the best performance for small ensemble sizes, while differences among the proposed mechanisms tends to be negligible as the ensemble size is greater than 5 . A similar behavior can be also seen in Fig. 1(f) where the performance for FNR 1\% are shown. A different behavior can be seen in Fig.s1(c)-(e), where the working point is set to 0\% FPR or FNR respectively. In these cases, $\Delta$-voting provides the worst performance, while the DLC and SDI-mean outperform the Mean-rule for any ensemble size in the case of FNR 0\%, while in the case of FPR 0\% performance improvements are shown for ensemble sizes greater than or equal to 5 . Thus, we can conclude that the proposed mechanisms allows exploiting the complementarity of different classifiers, especially in the case of large ensemble size.

In particular, in the case of the dataset at hand, we observed that the DLC and SDI-mean outperform all other techniques in any performance measure for ensembles size greater than or equal to 5 . The inspection of the values in the Table 1 clearly shows that the AUC does not allow to see any significant difference among the considered combination mechanisms. On the other hand, the values related to the operating point related to very low error rates, show the effectiveness of the proposed mechanism. This effectiveness has been also validated by performing the t-test with a $95 \%$ confidence on the difference in performance with the Mean-rule. All the differences, except those marked with a circle, are statistically significant. In addition, it is worth noting that in security applications even small differences in performances are of great value. 
The reported results allow to conclude that the proposed DLC and SDI-mean techniques based on the Score Decidability Index allows exploiting effectively the complementarity among different classifiers. In addition, depending on the performance measure of interest, the other two techniques based on a simplified combination can also provide good performances. In conclusion, it can be pointed out that the proposed Index provide an useful measure for the estimation of the parameters for combining an ensemble of two-class classifiers.

\section{References}

1. Fawcett, T.: An introduction to ROC Analysis. Pattern Recognition Letters 27(8), 861-874 (2006)

2. Fiérrez-Aguilar, J., Chen, Y., Ortega-Garcia, J., Jain, A.K.: Incorporating Image Quality in Multi-algorithm Fingerprint Verification. In: Zhang, D., Jain, A.K. (eds.) ICB 2005. LNCS, vol. 3832, pp. 213-220. Springer, Heidelberg (2005)

3. Fumera, G., Roli, F.: A theoretical and experimental analysis of linear combiners for multiple classifier systems. IEEE Transactions on Pattern Analysis and Machine Intelligence 27, 942-956 (2005)

4. Hanley, J.A., McNeil, B.J.: The meaning and the use of the area under a receiver operanting charateristic curve. Radiology 143, 29-36 (1982)

5. Huang, J., Ling, C.: Using AUC and Accuracy in Evaluating Learning Algorithms. IEEE Transactions on Knowledge and Data Engineering 17, 299-310 (2005)

6. Kittler, J., Hatef, M., Duin, R., Matas, J.: On combing classifiers. IEEE Trans. on PAMI 20(3), 226-239 (1998)

7. Kuncheva, L.: Combining Pattern Classifiers: Methods and Algorithms. John Wiley \& Sons Inc., Chichester (2004)

8. Marcialis, G.L., Roli, F.: Fusion of multiple fingerprint matchers by single layer perceptron with class-separation loss function. Pattern Recognition Letters 26, 1830-1839 (2005)

9. Nandakumar, K., Jain, A., Dass, S.: Quality-based Score Level Fusion in Multibiometric Systems. In: ICPR 2006, pp. 473-476 (2006)

10. Poh, N., Bengio, S.: Improving Fusion with Margin-Derived Confidence In Biometric Authentication Tasks. In: Kanade, T., Jain, A., Ratha, N.K. (eds.) AVBPA 2005. LNCS, vol. 3546, pp. 474-483. Springer, Heidelberg (2005)

11. Poh, N., Bengio, S.: Database, protocols and tools for evaluating score-level fusion algorithms in biometric authentication. Pattern Recognition 39(2), 223-233 (2006)

12. Ross, A.A., Nandakumar, K., Jain, A.K.: Handbook of multibiometrics. Springer, Heidelberg (2006)

13. Tronci, R., Giacinto, G., Roli, F.: Dynamic Score Combination: A Supervised and Unsupervised Score Combination. In: Perner, P. (ed.) MLDM 2009. LNCS (LNAI), vol. 5632, pp. 163-177. Springer, Heidelberg (2009)

14. Tumer, K., Gosh, J.: Linear and order statistics combiners for pattern classification. In: Combining Artificial Neural Nets, pp. 127-162. Springer, Heidelberg (1999)

15. Marrocco, C., Duin, R.P.W., Tortorella, F.: Maximizing the area under the ROC curve by pairwise feature combination. Pattern Recognition 41(6), 1961-1974 (2008)

16. Bigun, E., Bigun, J., Duc, B., Fischer, S.: Expert conciliation for multi modal person authentication systems by Bayesian statistics. In: Bigün, J., Borgefors, G., Chollet, G. (eds.) AVBPA 1997. LNCS, vol. 1206, pp. 291-300. Springer, Heidelberg (1997) 